\title{
Deterministic entanglement purification and complete nonlocal Bell-state analysis with hyperentanglement*
}

\author{
Yu-Bo Sheng ${ }^{1,2,3}$ and Fu-Guo Deng ${ }^{\dagger}$ \\ 1 Department of Physics, Beijing Normal University, Beijing 100875, People's Republic of China \\ ${ }^{2}$ College of Nuclear Science and Technology, Beijing Normal University, Beijing 100875, People's Republic of China \\ ${ }^{3}$ Key Laboratory of Beam Technology and Material Modification of Ministry of Education, \\ Beijing Normal University, Beijing 100875, People's Republic of China
}

(Dated: September 23, 2018)

\begin{abstract}
Entanglement purification is a very important element for long-distance quantum communication. Different from all the existing entanglement purification protocols (EPPs) in which two parties can only obtain some quantum systems in a mixed entangled state with a higher fidelity probabilistically by consuming quantum resources exponentially, here we present a deterministic EPP with hyperentanglement. Using this protocl, the two parties can, in principle, obtain deterministically maximally entangled pure states in polarization without destroying any less-entangled photon pair, which will improve the efficiency of long-distance quantum communication exponentially. Meanwhile, it will be shown that this EPP can be used to complete nonlocal Bell-state analysis perfectly. We also discuss this EPP in a practical transmission.
\end{abstract}

\section{INTRODUCTION}

The realization of long-distance quantum communication schemes should resort to the distribution of entangled states between distant locations [1]. Although photons are the optimal quantum information carriers in long-distance quantum communication as the interaction between them and environment is weaker than others, the polarization degree of freedom of photons is incident to the noise in a quantum channel. Noise will degrade the entanglement of a photon pair or even turn it into a mixed state. If the destructive effect of the noise is low, the two parties in quantum communication, say Alice and Bob, can first exploit entanglement purification to improve the entanglement of the quantum systems, and then achieve the goal of quantum communication with maximally entangled states. Entanglement purification becomes a very important element in quantum repeater [2] for long-distance quantum communication.

In 1996, Bennett et al. 3] proposed an entanglement purification protocol (EPP) based on quantum controlled-NOT (CNOT) logic operations, and subsequently it was improved by Deutsch et al. [4] using similar logic operations. In 2001, Pan et al. [5] proposed an EPP with linear optical elements. In 2002, Simon and Pan [6] improved their protocol. They considered a currently available source, a parametric down-conversion (PDC) source, to prepare entangled photon pairs, and they first used spatial entanglement to purify polarization entanglement. Both of these protocols should resort to sophisticated single-photon detectors, which is not a simple task in linear optics. In 2008, an EPP based on nondestructive quantum nondemolition detectors was proposed [7]. By far, all existing EPPs cannot obtain

\footnotetext{
*Published in Phys. Rev. A 81, 032307 (2010)

${ }^{\dagger}$ Corresponding author:fgdeng@bnu.edu.cn.
}

maximally entangled states. They only improve the fidelity of an ensemble in a mixed entangled state. In order to obtain some entangled states with higher fidelity, they have to consume more and more less-entanglement ones. Theoretically speaking, it is impossible to get a pair of photons in a maximally entangled state with conventional EPPs [3 3 , 7 .

Recently, the applications of hyperentangled states have been studied by some groups. A state of being simultaneously entangled in multiple degrees of freedom is called "a hyperentangled state" [8-10]. The most important use of hyperentanglements is in a complete deterministic local Bell-state analysis $11-13$. In 2008, with the help of the hyperentangled state in both polarization and orbit angular momentum, Barreiro et al. [14] beat the channel capacity limit for linear photonic superdense coding. With a type-I and type-II $\beta$ barium borate $(\mathrm{BBO})$ crystal, photon pairs produced by spontaneous parametric down-conversion (SPDC) can be in the hyperentangled state in polarization and spatial degrees of freedom [6] polarization, spatial, energy, and time degrees of freedom 8], polarization and frequency degrees of freedom [15]; and so on. In 2009, Vallone et. al. [16] also reported their experiment with a six-qubit hyperentangled state in three degrees of freedom. If we substitute the SPDC source in Ref.[15] for the PDC source in Ref.[6], we can produce the hyperentanglement with the following form:

$$
\frac{1}{2 \sqrt{2}}(|H H\rangle+|V V\rangle) \cdot\left(\left|\omega_{1} \omega_{2}\right\rangle+\left|\omega_{2} \omega_{1}\right\rangle\right) \cdot\left(\left|a_{1} b_{1}\right\rangle+\left|a_{2} b_{2}\right\rangle\right) .
$$

Here, $H(V)$ represents the horizontal (vertical) photon polarization, $\omega_{1}\left(\omega_{2}\right)$ represents the frequency of the signal (idler) photon, and $a_{1} b_{1}\left(a_{2} b_{2}\right)$ represents the spatial mode of photons.

In this article, we will present a deterministic EPP with hyperentangled states in the form of Eq.(1). The two 
parties in quantum communication, say Alice and Bob, can get a maximally entangled photon pair from each hyperentangled state in this EPP, which is, in essence, different from all the existing conventional EPPs 3 -7]. The deterministic feature of our protocol will improve the efficiency of long-distance quantum communication exponentially as the conventional EPPs will consume entangled quantum resources exponentially for obtaining some maximally entangled states. Also, this EPP can accomplish the complete nonlocal Bell-state analysis.

The article is organized as follows. In Sec.II A, we describe the principle of the deterministic entanglement purification for bit-flip errors with spatial entanglement. In Sec.II B, the purification for phase-flip errors is discussed. In Sec.III, we discuss the method of nonlocal Bell-state analysis with hyperentanglement. In Sec.IV, we analyze the essence of entanglement purification. In Sec.V, we discuss the present EPP in a practical transmission. A discussion and a summary are given in Sec.VI.

\section{DETERMINISTIC ENTANGLEMENT PURIFICATION}

It is well known that the most important application of EPPs is in constructing quantum repeaters for long-distance quantum communication in a noisy channel 2, 17 20]. In order to connect the adjacent nodes, the two parties in quantum communication should first transmit their photons in a noisy channel and then connect them with quantum entanglement swapping. Usually, the channel noise will degrade the entanglement of photon pairs. Also, the imperfect operations will disturb the entanglement of quantum systems. Now, let us start the explanation of our entanglement purification scheme by discussing an ordinary example. During a quantum-signal transmission, polarization degree of freedom suffers from the channel noise as both the spatial degree of freedom and the frequency degree of freedom are more stable than polarization. The previous experiments showed that the polarization entanglement is quite unsuitable for transmission over distances of more than a few kilometers in an optical fiber [1]. For example, Naik et al. demonstrated the Ekert protocol 21] over only a few meters [1, 22]. Also, they observed the quantum bit error rate (QBER) increase to $33 \%$ in the experimental implementation of the six-state protocol 23, 24]. For frequency coding [25 30], for example, the Besancon group performed a key distribution over a $20-\mathrm{km}$ single-mode optical-fiber spool. They recorded a $\mathrm{QBER}_{\text {opt }}$ contribution of approximately $4 \%$, and estimated that $2 \%$ could be attributed to the transmission of the central frequency by the Fabry-Perot cavity [30]. The experiment by Miná $\breve{r}$ et al. [31] for phase-noise measurements showed that in a realistic environment, the phase in long fibers (several tens of $\mathrm{km}$ ) remains stable, which is an acceptable level for time on the order of $100 \mu s$. The phase stabilization is relevant for the quantum repeaters in installed opti- cal fiber networks. In Simon's protocol [6], they also performed an EPP using spatial entanglement to purify polarization entanglement based on the good mode overlap on the PBS and phase stability that were achieved in previous experiments.

In fact, that frequency and spatial entanglement absolutely do not suffer from the noise is only a hypothesis and is unpractical. Here we only use it to show the basic principle for our entanglement purification process. We also will discuss the entanglement purification under a realistic environment. Moreover, other degrees of freedom, such as time-bin, which is more robust than polarization, can also be used to implement this scheme [32, 33]. In the discussion section of this article, we will show that the entanglement purification essentially performs entanglement transformation between different degrees of freedom, that is, transforms robust entanglement in channel transmissions (frequency and spatial) into easily manipulatable entanglements (polarization).

Under the hypothesis mentioned previously, the entanglement purification in the present scheme is divided into two steps, that is, purification for bit-flip errors and that for phase-flip errors. We discuss them in detail in this section as follows.

\section{A. Deterministic purification for bit-flip errors}

After the transmission, the hyperentangled state of Eq.(11) will become a mixed one in polarization:

$$
\begin{aligned}
\rho_{p} & =a\left|\Phi^{+}\right\rangle_{A B}\left\langle\Phi^{+}|+b| \Phi^{-}\right\rangle_{A B}\left\langle\Phi^{-}\right| \\
& +c\left|\Psi^{+}\right\rangle_{A B}\left\langle\Psi^{+}|+d| \Psi^{-}\right\rangle_{A B}\left\langle\Psi^{-}\right| .
\end{aligned}
$$

Here $a+b+c+d=1$ and $\rho_{p}$ is the mixed part of Eq.(1) in polarization. $\left|\Phi^{ \pm}\right\rangle_{A B}$ and $\left|\Psi^{ \pm}\right\rangle_{A B}$ are the four Bell states for an entangled photon pair $A B$ :

$$
\begin{aligned}
\left|\Phi^{ \pm}\right\rangle_{A B} & =\frac{1}{\sqrt{2}}\left(|H\rangle_{A}|H\rangle_{B} \pm|V\rangle_{A}|V\rangle_{B}\right), \\
\left|\Psi^{ \pm}\right\rangle_{A B} & =\frac{1}{\sqrt{2}}\left(|H\rangle_{A}|V\rangle_{B} \pm|V\rangle_{A}|H\rangle_{B}\right) .
\end{aligned}
$$

After the transmission, the initial state becomes

$$
\rho=\rho_{p} \rho_{f} \rho_{s}
$$

with one photon belonging to Alice and the other belonging to Bob. Here $\rho_{f}=\frac{1}{2}\left(\left|\omega_{1} \omega_{2}\right\rangle+\left|\omega_{2} \omega_{1}\right\rangle\right)\left(\left\langle\omega_{1} \omega_{2}\right|+\right.$ $\left.\left\langle\omega_{2} \omega_{1}\right|\right)$ and $\rho_{s}=\frac{1}{2}\left(\left|a_{1} b_{1}\right\rangle+\left|a_{2} b_{2}\right\rangle\right)\left(\left\langle a_{1} b_{1}\right|+\left\langle a_{2} b_{2}\right|\right)$. We also let $\left|\Psi_{f}\right\rangle=\frac{1}{\sqrt{2}}\left(\left|\omega_{1} \omega_{2}\right\rangle+\left|\omega_{2} \omega_{1}\right\rangle\right)$ and $\left|\Phi_{s}\right\rangle=$ $\frac{1}{\sqrt{2}}\left(\left|a_{1} b_{1}\right\rangle+\left|a_{2} b_{2}\right\rangle\right)$.

There are admixtures of the unwanted states $\left|\Phi^{-}\right\rangle_{A B}$ and $\left|\Psi^{ \pm}\right\rangle_{A B}$. We note that the state $\left|\Phi^{+}\right\rangle_{A B}$ becoming $\left|\Psi^{+}\right\rangle_{A B}$ is a bit-flip error, becoming $\left|\Phi^{-}\right\rangle_{A B}$ is a phaseflip error, and both a bit-flip error and a phase-flip error take place when $\left|\Phi^{+}\right\rangle_{A B}$ becomes $\left|\Psi^{-}\right\rangle_{A B}$. From Eqs.(2) and (5), the original state can be seen as a probabilistic 


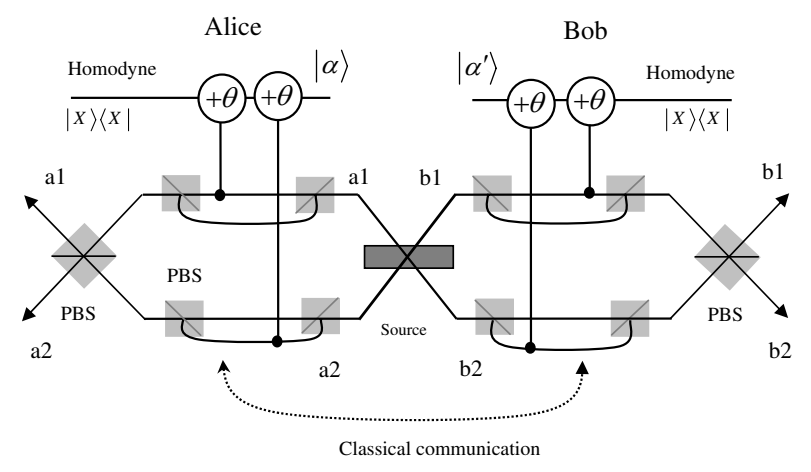

FIG. 1: Schematic drawing of the principle of bit-flip error purification. The source emits the entangled pair with the form of Eq.(1). One member of pair has been sent to Alice and the other to Bob. Both Alice and Bob perform X homodyne measurements on their coherent beams $|\alpha\rangle$ and $\left|\alpha^{\prime}\right\rangle$, respectively, and compare the results via classical communication. If their results are different, they need to perform a bit-flipping operation to correct this error. Otherwise, there are no bit-flip errors. PBS: polarizing beam splitter.

mixture of four pure states: with a probability of $a$ the photon pair in the state $\left|\Phi^{+}\right\rangle\left|\Psi_{f}\right\rangle\left|\Phi_{s}\right\rangle$, with a probability of $b$ the pair in the state of $\left|\Phi^{-}\right\rangle\left|\Psi_{f}\right\rangle\left|\Phi_{s}\right\rangle$, with the probability of $c$ and $d$ in $\left|\Psi^{ \pm}\right\rangle\left|\Psi_{f}\right\rangle\left|\Phi_{s}\right\rangle$. The whole task of purification is to correct the bit-flip and the phaseflip errors. So this scheme includes two steps, one for bit-flip error correction and the other for phase-flip error correction.

The principle of our scheme for bit-flip error correction is shown in Fig. 1, where $+\theta$ represents a crossKerr nonlinear medium which will make the coherent state $|\alpha\rangle$ pick up a phase shift $\theta$ when it and one photon couple with the medium [34 36]. We now consider the combinations $\left|\Phi^{+}\right\rangle\left|\Psi_{f}\right\rangle\left|\Phi_{s}\right\rangle$ and $\left|\Psi^{+}\right\rangle\left|\Psi_{f}\right\rangle\left|\Phi_{s}\right\rangle$. Let us first discuss the state $\left|\Phi^{+}\right\rangle\left|\Psi_{f}\right\rangle\left|\Phi_{s}\right\rangle$. In Fig. 1, the items $|H H\rangle\left(\left|\omega_{1} \omega_{2}\right\rangle+\left|\omega_{2} \omega_{1}\right\rangle\right)\left|a_{1} b_{1}\right\rangle$ and $|V V\rangle\left(\left|\omega_{1} \omega_{2}\right\rangle+\right.$ $\left.\left|\omega_{2} \omega_{1}\right\rangle\right)\left|a_{2} b_{2}\right\rangle$ make the two coherent beams $|\alpha\rangle$ and $|\alpha\rangle^{\prime}$ obtain the same phase shift of $\theta$, which can be detected by Alice and Bob with an $X$ homodyne measurement [34 36]. Finally, coupled by the two polarizing beam splitters (PBSs), they will emit from $a_{2} b_{2}$. The whole state becomes $\frac{1}{2}(|H H\rangle+|V V\rangle)\left(\left|\omega_{1} \omega_{2}\right\rangle+\left|\omega_{2} \omega_{1}\right\rangle\right)$. Following by the same principle, we can also get the state $\frac{1}{2}(|H H\rangle+|V V\rangle)\left(\left|\omega_{1} \omega_{2}\right\rangle+\left|\omega_{2} \omega_{1}\right\rangle\right)$ from $a_{1} b_{1}$ if both Alice and Bob get no phase shifts. In the case of $\left|\Psi^{+}\right\rangle\left|\Psi_{f}\right\rangle\left|\Phi_{s}\right\rangle$, it never leads to the same phase-shift case. If Alice gets the phase shift of $\theta$ and Bob gets no phase shift, it means that the proceeding state is changed to $\frac{1}{2}(|H V\rangle+$ $|V H\rangle)\left(\left|\omega_{1} \omega_{2}\right\rangle+\left|\omega_{2} \omega_{1}\right\rangle\right)$. The photon which belongs to Alice is in the mode of $a_{2}$, and which belongs to Bob is in $b_{1}$. There is another case for Alice and Bob. That is, Alice gets no phase shift and Bob gets $\theta$. The corresponding state is also $\frac{1}{2}(|H V\rangle+|V H\rangle) \cdot\left(\left|\omega_{1} \omega_{2}\right\rangle+\left|\omega_{2} \omega_{1}\right\rangle\right)$, but with the spatial modes of $a_{1}$ and $b_{2}$.

By applying our purification procedure, Alice and Bob can easily check the bit-flip error as they get different phase shifts with their $X$ homodyne measurements on their coherent beams. The spatial modes are also different, corresponding to the different collapsed states , but which can be completely determined. Therefore, by classical communication, if a bit-flip error occurs, Alice and Bob will get rid of the bit-flip error by performing a bit-flip operation $\sigma_{x}=|H\rangle\langle V|+| V\rangle\langle H|$. Next we show our protocol works for the other cases. The case of $\left|\Phi^{-}\right\rangle\left|\Psi_{f}\right\rangle\left|\Phi_{s}\right\rangle$ will get the same result with $\left|\Phi^{+}\right\rangle\left|\Psi_{f}\right\rangle\left|\Phi_{s}\right\rangle$. After the PBSs, the two photons will be either in the upper modes $a_{1}$ and $b_{1}$ or in the lower modes $a_{2}$ and $b_{2}$. For the case $\left|\Psi^{-}\right\rangle\left|\Psi_{f}\right\rangle\left|\Phi_{s}\right\rangle$, part of polarization both contains a bit-flip error and a phase-flip error, so Alice and Bob will get the phase shift of $\theta$ and 0 or 0 and $\theta$, respectively. It has an analogy with the case of $\left|\Psi^{+}\right\rangle\left|\Psi_{f}\right\rangle\left|\Phi_{s}\right\rangle$. We can also perform a bit-flip operation to correct it. In this case, a phase-flip error still remains.

\section{B. Deterministic purification for phase-flip errors}

So far, we have been talking about a bit-flip error for the mixed state in polarization. By correcting this error, the initial state in polarization becomes

$$
\rho_{p^{\prime}}=(a+c)\left|\Phi^{+}\right\rangle_{A B}\left\langle\Phi^{+}|+(b+d)| \Phi^{-}\right\rangle_{A B}\left\langle\Phi^{-}\right| .(6)
$$

As we know, a phase-flip error cannot be purified directly, but it can be transformed into a bit-flip error by Hadamard (H) operations. In an optical system, it can be finished by a $\lambda / 4$-wave plate (QWP). By performing the $\mathrm{H}$ operations on the two photons with two QWPs, Eq.(6) evolves:

$$
\rho_{p^{\prime \prime}}=(a+c)\left|\Phi^{+}\right\rangle_{A B}\left\langle\Phi^{+}|+(b+d)| \Psi^{+}\right\rangle_{A B}\left\langle\Psi^{+}\right| .(7)
$$

That is, the initial state becomes

$$
\rho^{\prime}=\rho_{p^{\prime \prime}} \rho_{f}
$$

It is interesting to find that the entanglement in frequency was not affected during the procedure discussed previously, but the spatial entanglement is consumed for correcting bit-flip errors.

Now we focus on the second step of our EPP: correcting the phase-flip error. In Fig. 2, two photons in the from of Eq. (8) belong to Alice and Bob, respectively. Two polarization-independent wavelength division multiplexers (WDMs) are used to guide photons to different paths, according to their frequencies. For example, in Alice's laboratory, it leads the photon in $\omega_{1}$ to the mode $c_{1}$ and the photon in $\omega_{2}$ to the mode $c_{2}$. However, in Bob's laboratory, it leads the photon in $\omega_{1}$ to the mode $d_{2}$ and the photon in $\omega_{2}$ to the mode $d_{1}$.

From Eq.(7), it follows that the original state of the pairs can be seen as a probabilistic mixture of two pure states: with a probability of $a+c$ the photon pair is in the state $\left|\Phi^{+}\right\rangle\left|\Psi_{f}\right\rangle$ and with a probability of 


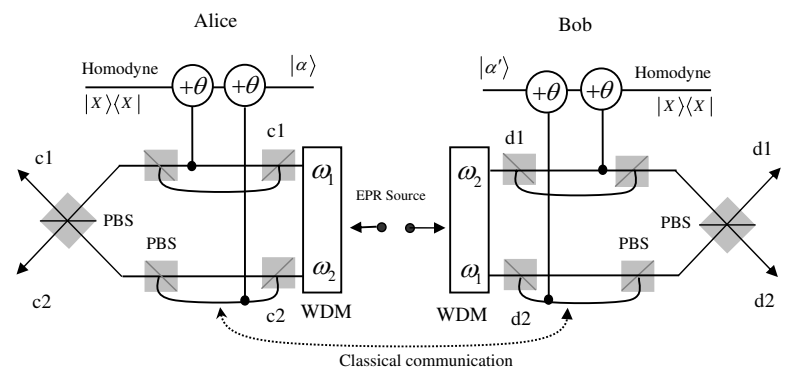

FIG. 2: Scheme showing the principle of phase-flip purification. After two Hadamard operations, a phase-flip error is transformed into a bit-flip error. Two WDMs are used to guide the the photons to the different paths according to their frequencies. Similar to the bit-flip error correction, if Alice and Bob get different phase shifts, the phase-flip error occurs, and then they add a bit-flipping operation to correct this error. Otherwise, there is no phase-flip error.

$b+d$ the pair is in the state $\left|\Psi^{+}\right\rangle\left|\Psi_{f}\right\rangle$. It is obvious that $\left|\Phi^{+}\right\rangle\left|\Psi_{f}\right\rangle$ leads to the same phase shift for both $\theta$ and 0 . They will be either in the mode $c_{1} d_{1}$ with the state of $\frac{1}{\sqrt{2}}\left(\left|H \omega_{2}\right\rangle_{A}\left|H \omega_{1}\right\rangle_{B}+\left|V \omega_{1}\right\rangle_{A}\left|V \omega_{2}\right\rangle_{B}\right)$ or in the mode $c_{2} d_{2}$ with the state of $\frac{1}{\sqrt{2}}\left(\left|H \omega_{1}\right\rangle_{A}\left|H \omega_{2}\right\rangle_{B}+\right.$ $\left.\left|V \omega_{2}\right\rangle_{A}\left|V \omega_{1}\right\rangle_{B}\right)$. This is the maximally entangled state we need. However, for $\left|\Psi^{+}\right\rangle\left|\Psi_{f}\right\rangle$, it never leads to the same phase shift. Alice will get $\theta$ while Bob will get 0 with the state $\frac{1}{\sqrt{2}}\left(\left|H \omega_{1}\right\rangle_{A}\left|V \omega_{2}\right\rangle_{B}+\left|V \omega_{2}\right\rangle_{A}\left|H \omega_{1}\right\rangle_{B}\right)$ in the mode $c_{2} d_{1}$ or Alice gets 0 and Bob gets $\theta$ with the state $\frac{1}{\sqrt{2}}\left(\left|H \omega_{2}\right\rangle_{A}\left|V \omega_{1}\right\rangle_{B}+\left|V \omega_{1}\right\rangle_{A}\left|H \omega_{2}\right\rangle_{B}\right)$ in the mode $c_{1} d_{2}$. We can perform a bit-flip operation to get rid of the errors. Finally, we will get the entangled state $\frac{1}{\sqrt{2}}\left(\left|H \omega_{2}\right\rangle_{A}\left|H \omega_{1}\right\rangle_{B}+\left|V \omega_{1}\right\rangle_{A}\left|V \omega_{2}\right\rangle_{B}\right)$ or $\frac{1}{\sqrt{2}}\left(\left|H \omega_{1}\right\rangle_{A}\left|H \omega_{2}\right\rangle_{B}+\left|V \omega_{2}\right\rangle_{A}\left|V \omega_{1}\right\rangle_{B}\right)$ with a deterministic spatial mode. With quantum frequency upconversion, we can erase distinguishability for frequency [37].

\section{NONLOCAL BELL-STATE ANALYSIS}

Now let us discuss the relationship between this entanglement purification protocol and a nonlocal Bell-state analysis (NBSA). A universal conclusion is that a completely local Bell-state analysis with linear optics is not possible and one can get an optimal success probability of only $\frac{3}{4}[38-40]$. Several works have shown that with additional degrees of freedom such as timing and momentum it is possible to distinguish the four Bell states locally [11 13]. However, compared with local BSA, NBSA cannot be completed with the collective operations and they can only resort to local operation and classical communication (LOCC). Here we will show that with hyperentangled states and quantum nondemolition measurement (QND), we can also perform a complete NBSA. The difference is that we have to need another two de- grees of freedom in NBSA, but only one is needed in local BSA. The initial state is a hyperentangled state with the form $\left|\Phi^{ \pm}\right\rangle\left|\Psi_{f}\right\rangle\left|\Phi_{s}\right\rangle$ or $\left|\Psi^{ \pm}\right\rangle\left|\Psi_{f}\right\rangle\left|\Phi_{s}\right\rangle$. The whole protocol is the same as our EPP discussed previously. The first step for NBSA is shown in Fig. 1. If Alice and Bob get the same phase shift, that is, if both get 0 or $\theta$, they can decide that the photon pair should be in one of the two states $\left|\Phi^{ \pm}\right\rangle$. Subsequently, they add a $\mathrm{H}$ operation on each photon and then make the second check of the phase shifts (shown in Fig. 2). If the phase shifts are still the same, the state should be $\left|\Phi^{+}\right\rangle$; otherwise, it is $\left|\Phi^{-}\right\rangle \cdot\left|\Psi^{ \pm}\right\rangle$can be distinguished in the same way. In the first step, if their phase shifts are different, it must be one of the two states $\left|\Psi^{ \pm}\right\rangle$. Alice and Bob perform a bitflip operation on one of the photons and a $\mathrm{H}$ operation on each photon, which will complete the transformation $\left|\Psi^{+}\right\rangle\left|\Psi_{f}\right\rangle \longrightarrow\left|\Phi^{+}\right\rangle\left|\Psi_{f}\right\rangle$ and $\left|\Psi^{-}\right\rangle\left|\Psi_{f}\right\rangle \longrightarrow\left|\Psi^{+}\right\rangle\left|\Psi_{f}\right\rangle$. In the second step, with the help of frequency degree of freedom, if the outcomes of the measurements on phase shifts are the same, Alice and Bob can conclude that the initial state should be $\left|\Psi^{+}\right\rangle$; otherwise, it is $\left|\Psi^{-}\right\rangle$.

\section{THE ESSENCE OF THE PRESENT ENTANGLEMENT PURIFICATION SCHEME}

In the previous works on entanglement purification, another entanglement of degree of freedom, such as spatial entanglement, has been used to purify the polarization entanglement of photon pairs [6, 7]. In Simon's protocol [6], the spatial entanglement can be used to purify a bitflip error. After consuming the resource of spatial entanglement, the phase-flip error has to be purified with the conventional method to repeat the same procedure 3 5. With another degree of freedom of photons, we can accomplish a deterministic entanglement purification.

Let us now discuss why our protocol can purify the mixed state completely. From Eq.(2), we know that there are two kinds of errors in the mixed state, that is, one is a bit-flip error and the other is a phase-flip error. The conventional EPPs [3-7] are used to purify a bit-flip error. The phase-flip error cannot be purified directly, but can be transformed into a bit-flip error. For the bit-flip purification, Alice and Bob can check whether one of the pairs has a bit-flip error. For instance, in Ref. [5], the error corresponds to the cross-combinations of $\left|\Phi^{+}\right\rangle_{A B}\left|\Psi^{+}\right\rangle_{A B}$ and $\left|\Psi^{+}\right\rangle_{A B}\left|\Phi^{+}\right\rangle_{A B}$. However, there always exists another possibility that both of the states have bit-flip errors, which corresponds to $\left|\Psi^{+}\right\rangle_{A B}\left|\Psi^{+}\right\rangle_{A B}$. In this case, Alice and Bob cannot pick up these corrupt states, and always keep them in the quantum systems that remain for a next purification. That is, a bit-flip error cannot be purified completely. Neither can a phase-flip error. They cannot make the remaining ensemble reach an indeed pure state. In Simon's protocol [6], they revealed that another kind of entanglement can also be used to purify the polarization entanglement state. In their protocol, they can correct the bit-flip error completely as the spa- 
tial entanglement state is a maximally entangled perfect pure state and is not effected by the channel noise. Their protocol does not lead to the case that each two-photon pair has bit-flip errors after their purification. Following the same principle, we use the frequency degree of freedom for a phase-flip error correction. Also, we can use another kind of degree of freedom to complete this task if it does not suffer from the channel noise.

LOCC cannot increase the entanglement of quantum systems. Therefore, the process of entanglement purification is essentially the transformation of entanglement. In the previous works [3-5], the transformation is between the same kind of entanglement, that is, polarization entanglement. So they need to consume the less-entangled pairs largely. The previous work of Simon [6] and this protocol show that entanglement can be transformed between some different degrees of freedom. We let the initial state be a hyperentangled state, and it owns three kinds of degrees of freedom. During the purification step, we consume the entanglement in frequency and spatial degrees of freedom to get a pure polarization entangled state. Thus, the whole entanglement purification process does not need to consume the photon pairs but to consume other degrees of freedom of entanglement.

\section{ENTANGLEMENT PURIFICATION IN A PRACTICAL TRANSMISSION}

We have discussed our deterministic entanglement purification scheme in the case where there are two degrees of freedom that are insensitive to channel noise. We use the spatial freedom and the frequency freedom of photons as an example to describe the principle of our scheme. Of course, the main experimental requirement of this scheme is the phase stability if we use the spatial entanglement and the frequency entanglement to purify the polarization entanglement. This requirement may limit the distance of the quantum communication. However, this scheme can be adapted to the case of energytime entanglement, which would allow the two parties inquantum communication to be a father apart [6, 41].

Now, let us discuss the present entanglement purification scheme with a practical transmission based on the spatial entanglement and the frequency entanglement.

In a practical transmission for long-distance quantum communication, the relative phase between two different spatial modes is sensitive to path-length instabilities, which may be caused by the fiber length dispersion, or atmospheric fluctuation in a free-space transmission. In this way, not only might part of the polarization of the hyperentangled state become a mixed state shown in Eq.(2), the entanglement in spatial mode may become $\frac{1}{\sqrt{2}}\left(\left|a_{1} b_{1}\right\rangle+e^{i \Delta \phi_{s}}\left|a_{2} b_{2}\right\rangle\right)$ after transmission. The relative phase between the different spatial modes is denoted by $\Delta \phi_{s}=k \Delta x$. Here $k$ is the wave vector of the photons and $\Delta x$ is the path-length dispersion between the two spatial modes with $\Delta x=x_{a_{1} b_{1}}-x_{a_{2} b_{2}}$. That is to say, the spatial entanglement will pick up a phase shift $\Delta \phi_{s}$.

Approximatively, the frequency entanglement has similar features to the spatial entanglement. That is, it may become $\frac{1}{\sqrt{2}}\left(\left|\omega_{1} \omega_{2}\right\rangle+e^{i \Delta \phi_{f}}\left|\omega_{2} \omega_{1}\right\rangle\right)$ after transmission. Here $\Delta \phi_{f}$ is the phase dispersion coming from the different frequencies.

After a practical transmission, the initial state may become

$$
\rho^{\prime}=\rho_{p} \rho_{f}^{\prime} \rho_{s}^{\prime}
$$

where

$$
\rho_{f}^{\prime}=\frac{1}{2}\left(\left|\omega_{1} \omega_{2}\right\rangle+e^{i \Delta \phi_{f}}\left|\omega_{2} \omega_{1}\right\rangle\right)\left(\left\langle\omega_{1} \omega_{2}\right|+e^{-i \Delta \phi_{f}}\left\langle\omega_{2} \omega_{1}\right|\right)
$$

and

$$
\rho_{s}^{\prime}=\frac{1}{2}\left(\left|a_{1} b_{1}\right\rangle+e^{i \Delta \phi_{s}}\left|a_{2} b_{2}\right\rangle\right)\left(\left\langle a_{1} b_{1}\right|+e^{-i \Delta \phi_{s}}\left\langle a_{2} b_{2}\right|\right) .
$$

With the first step for the purification of bit errors (the same as the case where the entanglements in the spatial and the frequency degrees of freedom do not suffer from the phase fluctuation, as shown in Fig. 1), if both Alice and Bob get the phase shift $\theta$ on their coherent beams, the photon pair is in the state $\frac{1}{2}(|H H\rangle+$ $\left.e^{i \Delta \phi_{s}}|V V\rangle\right)\left(\left|\omega_{1} \omega_{2}\right\rangle+e^{i \Delta \phi_{f}}\left|\omega_{2} \omega_{1}\right\rangle\right)$ with the probability of $\frac{A}{2}$ and in the state $\frac{1}{2}\left(|H H\rangle-e^{i \Delta \phi_{s}}|V V\rangle\right)\left(\left|\omega_{1} \omega_{2}\right\rangle+\right.$ $\left.e^{i \Delta \phi_{f}}\left|\omega_{2} \omega_{1}\right\rangle\right)$ with the probability of $\frac{B}{2}$, and they will emit from the spatial modes $a_{2} b_{2}$. If both Alice and Bob get the phase shift 0 , the photon pair will emit from the spatial modes $a_{1} b_{1}$ and will be in the state $\frac{1}{2}\left(e^{i \Delta \phi_{s}}|H H\rangle+|V V\rangle\right)\left(\left|\omega_{1} \omega_{2}\right\rangle+e^{i \Delta \phi_{f}}\left|\omega_{2} \omega_{1}\right\rangle\right)$ with the probability of $\frac{A}{2}$ and in the state $\frac{1}{2}\left(e^{i \Delta \phi_{s}}|H H\rangle-\right.$ $|V V\rangle)\left(\left|\omega_{1} \omega_{2}\right\rangle+e^{i \Delta \phi_{f}}\left|\omega_{2} \omega_{1}\right\rangle\right)$ with the probability of $\frac{B}{2}$. When Alice gets the phase shift $\theta$ and Bob gets 0 , the photon pair will emit from $a_{2} b_{1}$ and will be in the state $\frac{1}{2}\left(|H V\rangle+e^{i \Delta \phi_{s}}|V H\rangle\right)\left(\left|\omega_{1} \omega_{2}\right\rangle+e^{i \Delta \phi_{f}}\left|\omega_{2} \omega_{1}\right\rangle\right)$ with the probability of $\frac{C}{2}$ and in the state $\frac{1}{2}\left(|H V\rangle-e^{i \Delta \phi_{s}}|V H\rangle\right)$. $\left(\left|\omega_{1} \omega_{2}\right\rangle+e^{i \Delta \phi_{f}}\left|\omega_{2} \omega_{1}\right\rangle\right)$ with the probability of $\frac{D}{2}$. When Alice gets the phase shift 0 and Bob gets $\theta$, the photon pair will emit from $a_{1} b_{2}$ and will be in the state $\frac{1}{2}\left(e^{i \Delta \phi_{s}}|H V\rangle+|V H\rangle\right)\left(\left|\omega_{1} \omega_{2}\right\rangle+e^{i \Delta \phi_{f}}\left|\omega_{2} \omega_{1}\right\rangle\right)$ with the probability of $\frac{C}{2}$ and in the state $\frac{1}{2}\left(e^{i \Delta \phi_{s}}|H V\rangle-|V H\rangle\right)$. $\left(\left|\omega_{1} \omega_{2}\right\rangle+e^{i \Delta \phi_{f}}\left|\omega_{2} \omega_{1}\right\rangle\right)$ with the probability of $\frac{D}{2}$. With some unitary operations, Alice and Bob can make the state of their photon pair in the polarization degree of freedom be

$$
\rho_{p^{\prime}}^{\prime}=(a+c)\left|\Phi^{\prime+}\right\rangle_{A B}\left\langle\Phi^{\prime+}|+(b+d)| \Phi^{\prime-}\right\rangle_{A B}\left\langle\Phi^{\prime-}\right| .
$$

Here

$$
\begin{aligned}
\left|\Phi^{\prime+}\right\rangle_{A B} & =\frac{1}{\sqrt{2}}\left(|H H\rangle+e^{i \Delta \phi_{s}}|V V\rangle\right), \\
\left|\Phi^{\prime-}\right\rangle_{A B} & =\frac{1}{\sqrt{2}}\left(|H H\rangle-e^{i \Delta \phi_{s}}|V V\rangle\right) .
\end{aligned}
$$


Also, the state $\rho_{p^{\prime}}^{\prime}$ can be rewritten under the basis $\left\{\left|\Phi^{+}\right\rangle,\left|\Phi^{-}\right\rangle,\left|\Psi^{+}\right\rangle,\left|\Psi^{-}\right\rangle\right\}$as

$$
\begin{aligned}
\rho_{p^{\prime}}^{\prime} & =\frac{1}{2}\left[1+(a+c-b-d) \cos \Delta \phi_{s}\right]\left|\Phi^{+}\right\rangle_{A B}\left\langle\Phi^{+}\right| \\
& +\frac{1}{2}\left[1-(a+c-b-d) \cos \Delta \phi_{s}\right]\left|\Phi^{-}\right\rangle_{A B}\left\langle\Phi^{-}\right| .
\end{aligned}
$$

That is, all the bit-flip errors in the photon pair are corrected completely and there are only phase-flip errors in the quantum system.

After the purification for bit-flip errors, Alice and Bob can transfer phase-flip errors into bit-flip errors with unitary operations again. That is, the state of the photon pair becomes

$$
\begin{aligned}
\rho_{p^{\prime}}^{\prime \prime} & =\frac{1}{2}\left[1+(a+c-b-d) \cos \Delta \phi_{s}\right]\left|\Phi^{+}\right\rangle_{A B}\left\langle\Phi^{+}\right| \\
& +\frac{1}{2}\left[1-(a+c-b-d) \cos \Delta \phi_{s}\right]\left|\Psi^{+}\right\rangle_{A B}\left\langle\Psi^{+}\right| .
\end{aligned}
$$

With the setup shown in Fig. 2, if both Alice and Bob get the phase shift $\theta$, the photon pair will emit from $c_{2} d_{2}$ and will be in the state $\frac{1}{\sqrt{2}}\left(\left|H \omega_{1}\right\rangle_{A}\left|H \omega_{2}\right\rangle_{B}+e^{i \Delta \phi_{f}}\left|V \omega_{2}\right\rangle_{A}\left|V \omega_{1}\right\rangle_{B}\right)$. If both Alice and Bob get the phase shift 0 , the photon pair will emit from $c_{1} d_{1}$ and will be in the state $\frac{1}{\sqrt{2}}\left(e^{i \Delta \phi_{f}}\left|H \omega_{1}\right\rangle_{A}\left|H \omega_{2}\right\rangle_{B}+\left|V \omega_{2}\right\rangle_{A}\left|V \omega_{1}\right\rangle_{B}\right)$. When Alice gets the phase shift $\theta$ and Bob gets 0 , the photon pair will emit from $c_{2} d_{1}$ and will be in the state $\frac{1}{\sqrt{2}}\left(\left|H \omega_{1}\right\rangle_{A}\left|V \omega_{2}\right\rangle_{B}+e^{i \Delta \phi_{f}}\left|V \omega_{2}\right\rangle_{A}\left|H \omega_{1}\right\rangle_{B}\right)$. When Alice gets the phase shift 0 and Bob gets $\theta$, the photon pair will emit from $c_{1} d_{2}$ and will be in the state $\frac{1}{\sqrt{2}}\left(e^{i \Delta \phi_{f}}\left|H \omega_{1}\right\rangle_{A}\left|V \omega_{2}\right\rangle_{B}+\left|V \omega_{2}\right\rangle_{A}\left|H \omega_{1}\right\rangle_{B}\right)$. With quantum frequency up-conversion, Alice and Bob can erase distinguishability for frequency [37] and they will get the entangled state $\frac{1}{\sqrt{2}}\left(|H\rangle_{A}|H\rangle_{B}+e^{i \Delta \phi_{f}}|V\rangle_{A}|V\rangle_{B}\right)$ with some unitary operations.

As the case discussed in Sec.II, Alice and Bob can correct the bit-flip errors in their photon pair completely. In the step for purification of phase-flip errors, the two different frequency modes will introduce a relative phase shift $\Delta \phi_{f}$ in the Bell state. In theory, $\Delta \phi_{f}$ does not change if the channel lengths do not fluctuate with time $t$. That is, Alice can get approximatively the maximally entangled state $\frac{1}{\sqrt{2}}\left(|H\rangle_{A}|H\rangle_{B}+e^{i \Delta \phi_{f}}|V\rangle_{A}|V\rangle_{B}\right)$. With a phase compensation, Alice and Bob will get the standard Bell state $\frac{1}{\sqrt{2}}\left(|H\rangle_{A}|H\rangle_{B}+|V\rangle_{A}|V\rangle_{B}\right)$.

\section{DISCUSSION AND SUMMARY}

There are two approximative methods used in our scheme in a practical transmission. One is the assumption that the phase dispersion in frequency degree of freedom $\Delta \phi_{f}$ is independent of that in spatial modes $\Delta \phi_{s}$.
The other is the invariability of $\Delta \phi_{f}$ with time $t$. Certainly, the case in a practical experiment is more complicated than that with these two assumptions.

For the state

$$
\begin{aligned}
|\Phi\rangle_{f s} \equiv & \frac{1}{2}\left(\left|\omega_{1} \omega_{2}\right\rangle+\left|\omega_{2} \omega_{1}\right\rangle\right) \cdot\left(\left|a_{1} b_{1}\right\rangle+\left|a_{2} b_{2}\right\rangle\right) \\
= & \frac{1}{2}\left(\left|\omega_{1} \omega_{2}\right\rangle\left|a_{1} b_{1}\right\rangle+\left|\omega_{2} \omega_{1}\right\rangle\left|a_{1} b_{1}\right\rangle\right. \\
& \left.+\left|\omega_{1} \omega_{2}\right\rangle\left|a_{2} b_{2}\right\rangle+\left|\omega_{2} \omega_{1}\right\rangle\left|a_{2} b_{2}\right\rangle\right)
\end{aligned}
$$

each term will pick up a relative phase in a practical transmission. That is, the state $|\Phi\rangle_{f s}$ will become

$$
\begin{aligned}
|\Phi\rangle_{f s}^{\prime}= & \frac{1}{2}\left[e^{\frac{i}{v}\left(\omega_{1} L_{a_{1}}+\omega_{2} L_{b_{1}}\right)}\left|\omega_{1} \omega_{2}\right\rangle\left|a_{1} b_{1}\right\rangle\right. \\
& +e^{\frac{i}{v}\left(\omega_{2} L_{a_{1}}+\omega_{1} L_{b_{1}}\right)}\left|\omega_{2} \omega_{1}\right\rangle\left|a_{1} b_{1}\right\rangle \\
& +e^{\frac{i}{v}\left(\omega_{1} L_{a_{2}}+\omega_{2} L_{b_{2}}\right)}\left|\omega_{1} \omega_{2}\right\rangle\left|a_{2} b_{2}\right\rangle \\
& \left.+e^{\frac{i}{v}\left(\omega_{2} L_{a_{2}}+\omega_{1} L_{b_{2}}\right)}\left|\omega_{2} \omega_{1}\right\rangle\left|a_{2} b_{2}\right\rangle\right] .
\end{aligned}
$$

Here $v$ is the velocity of photons in quantum channel. $L_{a_{1}}, L_{a_{2}}, L_{b_{1}}$, and $L_{b_{2}}$ are the channel lengths in the spatial modes $a_{1}, a_{2}, b_{1}$, and $b_{2}$, respectively. When

$$
\begin{aligned}
\omega_{2} L_{a_{2}}+\omega_{1} L_{b_{2}} \gg & \omega_{2} L_{a_{1}}+\omega_{1} L_{b_{1}}+\omega_{1}\left(L_{a_{2}}-L_{a_{1}}\right) \\
& +\omega_{2}\left(L_{b_{2}}-L_{b_{1}}\right)-\omega_{2} L_{a_{2}}-\omega_{1} L_{b_{2}}
\end{aligned}
$$

$|\Phi\rangle_{f s}^{\prime}$ can be rewritten as

$$
\begin{aligned}
|\Phi\rangle_{f s}^{\prime} \approx & \frac{1}{2}\left(e^{\frac{i}{v}\left(\omega_{1} L_{a_{1}}+\omega_{2} L_{b_{1}}\right)}\right. \\
& \times\left\{\left|\omega_{1} \omega_{2}\right\rangle+e^{\frac{i}{v}\left[\left(\omega_{2}-\omega_{1}\right) L_{a_{1}}+\left(\omega_{1}-\omega_{2}\right) L_{b_{1}}\right]}\left|\omega_{2} \omega_{1}\right\rangle\right\} \\
& \left.\times\left\{\left|a_{1} b_{1}\right\rangle+e^{\frac{i}{v}\left[\omega_{1}\left(L_{a_{2}}-L_{a_{1}}\right)+\omega_{2}\left(L_{b_{2}}-L_{b_{1}}\right)\right]}\left|a_{2} b_{2}\right\rangle\right\}\right) .
\end{aligned}
$$

That is, when $2\left(\omega_{2} L_{a_{2}}+\omega_{1} L_{b_{2}}\right)-\omega_{2} L_{a_{1}}-\omega_{1} L_{b_{1}}-$ $\omega_{1}\left(L_{a_{2}}-L_{a_{1}}\right)-\omega_{2}\left(L_{b_{2}}-L_{b_{1}}\right) \gg 0$, the phase dispersion in frequency degree of freedom $\Delta \phi_{f}$ can be regarded as being independent of that in spatial modes $\Delta \phi_{s}$.

If $\Delta \phi_{f}(t)$ fluctuates with time $t$ in a small region, Alice and Bob will get an ensemble in the state $\rho_{e}=$ $F_{f}\left|\Phi^{+}\right\rangle\left\langle\Phi^{+}\left|+\left(1-F_{f}\right)\right| \Phi^{-}\right\rangle\left\langle\Phi^{-}\right|$after a phase compensation $e^{i \Delta \phi_{f}(0)}$, and they can, in this case, purify this ensemble for getting some high-fidelity entangled states with conventional EPPs [3 5, 7]. Here $F_{f}=\frac{1}{2 T} \int_{0}^{T}(1+$ $\left.\cos \left(\Delta_{f}(t)\right)\right) \mathrm{d} t, 1-F_{f}=\frac{1}{2 T} \int_{0}^{T}\left(1-\cos \left(\Delta_{f}(t)\right)\right) \mathrm{d} t$, and $\left.\Delta_{f}(t)\right) \equiv \Delta \phi_{f}(t)-\Delta \phi_{f}(0)$. Different from the case with a fixed phase dispersion, Alice and Bob can only correct completely the bit-flip errors in the photon pair in this case. On the one hand, they will remove the phase-flip errors in the polarization degree of freedom. On the other hand, they will introduce the phase-flip errors in the frequency degree of freedom. The latter comes from the phase dispersion between the two frequencies of photons. If the latter is smaller than the former, this scheme can be used to depress the ratio of phase-flip errors. 
If $\Delta \phi_{f}(t)$ fluctuates acutely with time $t, F_{f} \approx \frac{1}{2}$ and the ensemble maybe become a completely mixed state and the two parties cannot distill maximally entangled states, the same as the conventional EPPs in the case that the initial fidelity is smaller than $\frac{1}{2}$. In fact, a quantum channel fluctuating with time $t$ acutely is unsuitable for entanglement purification as the Bell state required is mixed with other Bell states uniformly. As the spatial and frequency degrees of freedom of photons are more stable than polarization, the conventional EPPs will not work if the present scheme does not work.

In the process of describing the principle of our entanglement purification scheme, we exploit the cross-Kerr nonlinearity to construct the QNDs. In fact, we should acknowledge that, on the one hand, a clean cross-Kerr nonlinearity is quite a controversial assumption with current technology. As pointed out in Refs. [42 [44], the single-photon Kerr nonlinearity may do not help quantum computation, and a large phase shift via a "giant Kerr effect" with single-photon wave packets is impossible. On the other hand, here a cross-Kerr nonlinearity is only used to make a parity check for two photons and a strong Kerr nonlinearity is not required. Meanwhile, other elements can also be used to construct QNDs $45-$ 47] for this scheme.

In summary, we have presented a deterministic entanglement purification scheme for purifying an arbitrary mixed state in polarization with present technology. The biggest advantage of this scheme is that it works in a deterministic way in principle. That is, two parties can obtain a maximally entangled polarization state from each hyperentangled state, which will improve the efficiency of long-distance quantum communication exponentially. This protocol can also be used to do the complete nonlocal Bell-state analysis, which reveals that this deterministic entanglement purification scheme is equal to a complete nonlocal Bell-state analysis. In a practical transmission, this scheme can be used to correct all bit-flip errors approximatively and depress the phase-flip errors in the polarization degree of freedom, which will make it more efficient than conventional EPPs. We believe that this scheme might be very useful in the realization of long-distance quantum communication in the future as entanglement purification is a very important element in a quantum repeater and a quantum network.

\section{ACKNOWLEDGEMENTS}

This work is supported by the National Natural Science Foundation of China under Grant No. 10974020, the Foundation for the Author of National Excellent Doctoral Dissertation of P. R. China under Grant No. 200723, and the Beijing Natural Science Foundation under Grant No. 1082008.
[1] N. Gisin, G. Ribordy, W. Tittel, and H. Zbinden, Rev. Mod. Phys. 74, 145 (2002).

[2] H. J. Briegel, W. Dür, J. I. Cirac, and P. Zoller, Phys. Rev. Lett. 81, 5932 (1998).

[3] C. H. Bennett, G. Brassard, S. Popescu, B. Schumacher, J. A. Smolin, and W. K. Wootters, Phys. Rev. Lett. 76, 722 (1996).

[4] D. Deutsch, A. Ekert, R. Jozsa, C. Macchiavello, S. Popescu, and A. Sanpera, Phys. Rev. Lett. 77, 2818 (1996).

[5] J. W. Pan, C. Simon, and A. Zellinger, Nature 410, 1067 (2001); J. W. Pan, S. Gasparonl, R. Ursin, G. Weihs, and A. Zellinger, Nature (London) 423, 417 (2003).

[6] C. Simon and J. W. Pan, Phys. Rev. Lett. 89, 257901 (2002).

[7] Y. B. Sheng, F. G. Deng, and H. Y. Zhou, Phys. Rev. A 77, 042308 (2008).

[8] J. T. Barreiro, N. K.Langford, N. A. Peters, and P. G. Kwiat, Phys. Rev. Lett. 95, 260501 (2005).

[9] M. Barbieri, C.Cinelli, P. Mataloni, and F. DeMartini, Phys. Rev. A 72, 052110 (2005).

[10] S. P. Walborn, P. H. Souto Ribeiro, L. Davidovich, F. Mintert, and A. Buchleitner, Nature (London) 440, 1022 (2006).

[11] S. P. Walborn, S. Padua, and C. H. Monken, Phys. Rev. A 68, 042313 (2003).

[12] T. C. Wei, J. T. Barreiro, and P. G. Kwiat, Phys. Rev. A 75, 060305(R) (2007).

[13] C. Schuck, G. Huber, C. Kurtsiefer, and H. Weinfurter,
Phys. Rev. Lett. 96, 190501 (2006).

[14] J. T. Barreiro, T. C. Wei, and P. G. Kwiat, Nat. Phys. 4, 282 (2008).

[15] A. Yabushita and T. Kobayashi, Phys. Rev. A 69, 013806 (2004).

[16] G. Vallone, R. Ceccarelli, F. De Martini, and P. Mataloni, Phys. Rev. A 79, 030301(R) (2009).

[17] L. M. Duan, M. D. Lukin, J. T. Cirac, and P. Zoller, Nature(London) 414, 413 (2001).

[18] N. Sangouard, C. Simon, J. Miná $\breve{r}$, H. Zbinden, H. de Riedmatten and N. Gisin, Phys. Rev. A 76, 050301(R) (2007).

[19] C. Simon, H. de Riedmatten, M. Afzelius, N. Sangouard, H. Zbinden, and N. Gisin, Phys. Rev. Lett. 98, 190503 (2007).

[20] B. Zhao, Z. B. Chen, Y. A. Chen, J. Schmiedmayer, and J. W. Pan, Phys. Rev. Lett. 98, 240502 (2007).

[21] A. K. Ekert, Phys. Rev. Lett. 67, 661 (1991). (2007).

[22] D. S. Naik, C. G. Peterson, A. G. White, A. J. Berglund, and P. G. Kwiat, Phys. Rev. Lett. 84, 4733 (2000).

[23] D. Bruss, Phys. Rev. Lett. 81, 3018 (1998).

[24] H. Bechmann-Pasquinucci and N. Gisin, Phys. Rev. A 59, 4238 (1999).

[25] T. Zhang, Z. Q. Yin, T. F. Han, and G. C. Guo, Opt. Commun. 281, 4800 (2008).

[26] M. Bloch, S. W. McLaughlin, J. M. Merolla, and F. Patois, Opt. Lett. 32, 301 (2007).

[27] B. Huttner, N. Imoto, N. Gisin, and T. Mor,, Phys. Rev. 
A 51, 1863 (1995).

[28] P. C. Sun, Y. Mazurenko, and Y. Fainman, Opt. Lett. 20, 1062 (1995).

[29] Y. Mazurenko, R.Giust, and J. P. Goedgebuer,, Opt. Commun. 133, 87 (1997).

[30] J. M. Merolla, Y. Mazurenko, J. P. Goedgebuer, and W. T. Rhodes, Phys. Rev. Lett. 82, 1656 (1999).

[31] J. Miná $\breve{r}$, H. deRiedmatten, C. Simon, H. Zbinden, and N. Gisin, Phys. Rev. A 77, 052325 (2008).

[32] R. T. Thew, S. Tanzilli, W. Tittel, H. Zbinden and N. Gisin, Phys. Rev. A 66, 062304, (2002).

[33] I. Marcikic, H. deRiedmatten, W. Tittel, H. Zbinden, M. Legre, and N. Gisin, Phys. Rev. Lett. 93, 180502, (2004).

[34] K. Nemoto and W. J. Munro, Phys. Rev. Lett. 93, 250502 (2004).

[35] S. D. Barrett, P. Kok, K. Nemoto, R. G. Beausoleil, W. J. Munro, and T. P. Spiller, Phys. Rev. A 71, 060302(R) (2005).

[36] P. Kok, W. J. Munro, K. Nemoto, T. C. Ralph, J. P.
Dowling, and G. J. Milburn, Rev. Mod. Phys. 79, 135 (2007).

[37] H. Takesue, Phys. Rev. Lett. 101, 173901 (2008).

[38] L. Vaidman and N. Yoran, Phys. Rev. A 59, 116 (1999).

[39] N. Lutkenhaus, J. Calsamiglia, and K. A. Suominen, Phys. Rev. A 59, 3295 (1999).

[40] J. Calsamiglia, Phys. Rev. A 65, 030301(R) (2002).

[41] J. Brendel, N. Gisin, W. Tittel, and H. Zbinden, Phys. Rev. Lett. 82, 2594 (1999).

[42] J. H. Shapiro, Phys. Rev. A 73, 062305 (2006).

[43] J. H. Shapiro and M. Razavi, New J. Phys. 9, 16 (2007).

[44] J. G-Banacloche, arXiv:0911.4682 1 (2009).

[45] G. J. Pryde, J. L. O'Brien, A. G. White, S. D. Bartlett, and T.C. Ralph, Phys. Rev. Lett. 92, 190402 (2004)

[46] T. C. Ralph, S. D. Bartlett, J. L. O'Brien, G. J. Pryde, and H. M. Wiseman, Phys. Rev. A 73, 012113 (2006)

[47] G. J. Pryde, J. L. O'Brien, A. G. White, T. C. Ralph, and H. M. Wiseman, Phys. Rev. Lett. 94, 220405 (2005). 